\title{
Effect of leukotriene receptor antagonist therapy on the risk of asthma exacerbations in patients with mild to moderate asthma: an integrated analysis of zafirlukast trials
}

\author{
Neil C Barnes, Christopher J Miller
}

\begin{abstract}
Background-Asthma exacerbations contribute substantially to morbidity, and their reduction is an important therapeutic objective. In this integrated analysis the risk of asthma exacerbations was assessed during treatment with the leukotriene receptor antagonist zafirlukast. Methods-Data were collected from all five double blind, multicentre, randomised, placebo controlled, 13 week trials of zafirlukast $20 \mathrm{mg}$ twice daily performed in steroid-naive patients with mild to moderate asthma. Exacerbation data were collected prospectively during monitoring of adverse events and concomitant medication use. Pooled data were used to assess the relative risk of asthma exacerbations using three definitions: worsening of asthma leading to withdrawal from the study; requirement for additional antiasthma therapy (excluding increased short acting $\beta_{2}$ agonist use); and requirement for oral corticosteroid therapy.

Results-The proportion of patients with an asthma exacerbation leading to withdrawal was consistently lower in the group treated with zafirlukast $20 \mathrm{mg}$ twice daily than in the placebo group. Overall, the risk of an asthma exacerbation requiring withdrawal from zafirlukast therapy was approximately half that of placebo (odds ratio $0.45 ; 95 \% \mathrm{CI} 0.26$ to $0.76 ; p=0.003$ ). Similar results were observed for exacerbations requiring additional control medication (odds ratio $=0.47 ; 95 \%$ CI 0.30 to $0.74 ; p=0.001$ ) and oral corticosteroid rescue (odds ratio $=0.53 ; 95 \%$ CI 0.32 to $0.86 ; \mathrm{p}=0.010)$.
\end{abstract}

Department of Respiratory Medicine, The London Chest Hospital, London E2 9JX, UK

N C Barnes

AstraZeneca, Wilmington, Delaware, USA

C J Miller

Correspondence to: Dr N C Barnes

Received 6 May 1999 Returned to authors 18 June 1999

Revised manuscript received

10 February 2000

Accepted for publication

1 March 2000 patient improvement. These efficacy outcome measures are surrogate measures of disease control but are useful to physicians and patients for monitoring asthma. However, the outcome measures that have the greatest effect on patients are likely to be symptom reduction and prevention of asthma exacerbations, particularly those resulting in days absent from work or school or the need for emergency treatment or admission to hospital. ${ }^{1}$ The reduction of such exacerbations is increasingly recognised as an important goal of asthma control therapy. ${ }^{2}$ Consequently, exacerbations of asthma are now being used as efficacy outcome measures in clinical trials for the evaluation of new anti-asthma therapies.

At present, inhaled corticosteroids have been shown unequivocally to decrease the risk of asthma exacerbations $^{3-7}$ and these drugs represent the mainstay of anti-inflammatory treatment in asthma. In addition, evidence suggests that long acting $\beta_{2}$ agonists also provide protection from asthma exacerbations. ${ }^{7}$ However, drugs that interfere with the leukotriene pathway-that is, 5-lipoxygenase inhibitors and leukotriene receptor antagonists-may also prevent or reduce the severity of asthma exacerbations. The cysteinyl leukotrienes are important mediators of acute airway obstruction in asthma ${ }^{89}$ and increased urinary levels have been observed in patients during severe exacerbations. ${ }^{10}$

Compared with placebo, the leukotriene receptor antagonist zafirlukast has been shown to improve pulmonary function, reduce daytime and night time asthma symptoms, decrease the use of $\beta_{2}$ agonists as rescue medication, and decrease treatment failure rates. ${ }^{11-13}$ The objective of this analysis of data from all five randomised, double blind, placebo controlled, 13 week trials comparing the most widely used dosage of zafirlukast $(20 \mathrm{mg}$ twice daily) with placebo in steroid-naive patients with mild to moderate asthma was to determine the role of zafirlukast in reducing the risk of exacerbations that led to trial withdrawal or required additional treatment.

\section{Methods}

DATA SOURCES

We chose to integrate data from all randomised trials (all sponsored by Zeneca Pharmaceuticals, Wilmington, Delaware, USA and Zeneca Ltd, Alderley Park, UK) conducted to date that met the following criteria: design-13 week, double blind, parallel group; treatment- 
Table 1 Characteristics of patients in each of the five multicentre trials by treatment group

\begin{tabular}{|c|c|c|c|c|c|c|c|c|c|c|c|c|}
\hline & \multicolumn{2}{|l|}{ Trial $A$} & \multicolumn{2}{|l|}{ Trial B } & \multicolumn{2}{|l|}{ Trial C } & \multicolumn{2}{|l|}{ Trial D } & \multicolumn{2}{|l|}{ Trial E } & \multicolumn{2}{|c|}{ Integrated data set } \\
\hline & Zafirlukast & Placebo & Zafirlukast & Placebo & Zafirluka & tPlacebo & Zafirluka & t Placebo & Zafirlukast & Placebo & Zafirlukast & Placebo \\
\hline No. patients & 43 & 46 & 514 & 248 & 96 & 95 & 88 & 80 & 231 & 223 & 972 & 692 \\
\hline $\mathrm{M} / \mathrm{F}(\%)$ & $51 / 49$ & $54 / 46$ & $57 / 43$ & $59 / 41$ & $55 / 45$ & $53 / 47$ & $58 / 42$ & $49 / 51$ & $45 / 55$ & $41 / 59$ & $54 / 46$ & $51 / 49$ \\
\hline Mean (SD) age (years) & $35(11)$ & $33(13)$ & $31(12)$ & $31(13)$ & $32(14)$ & $30(13)$ & $34(13)$ & $33(12)$ & $33(14)$ & $32(13)$ & $32(13)$ & $32(13)$ \\
\hline Ethnic origin: & & & $450 / 24 /$ & $216 / 12 /$ & & & & & & $190 / 17 /$ & $853 / 51 /$ & $602 / 38 /$ \\
\hline $\begin{array}{l}\text { white/black/other } \\
\text { Mean (SD) FEV }\end{array}$ & $36 / 4 / 3$ & $38 / 5 / 3$ & 40 & 20 & $84 / 4 / 8$ & $78 / 4 / 13$ & $88 / 0 / 0$ & $80 / 0 / 0$ & $195 / 19 / 17$ & 16 & 68 & 52 \\
\hline Mean (SD) daytime & $14(10)$ & $I$ & & & & & 01 (1 & 02( & & 00 & & \\
\hline $\begin{array}{l}\text { Mean (SD) PEF variability } \\
(\%)^{\star} \ddagger\end{array}$ & $\begin{array}{l}14.4 \\
(11.2)\end{array}$ & $\begin{array}{l}13.2 \\
(10.4)\end{array}$ & $\begin{array}{l}1.0(0.4 \\
13.2 \\
(10.4)\end{array}$ & $\begin{array}{l}1.0(0.4) \\
11.7 \\
(10.2)\end{array}$ & $\begin{array}{l}1.7(0 \\
12.4 \\
(8.8)\end{array}$ & $\begin{array}{l}1.0(0.4 \\
14.2 \\
(12.7)\end{array}$ & $\begin{array}{l}9.1(0 \\
9.7 \\
(6.6)\end{array}$ & $\begin{array}{l}11.0 \\
(8.2)\end{array}$ & $\begin{array}{l}1.8(0 \\
3.5 \\
(9.7)\end{array}$ & $\begin{array}{l}1.9(0.4) \\
14.8 \\
(10.2)\end{array}$ & $\begin{array}{l}1.6(0 \\
12.9 \\
(9.9)\end{array}$ & $\begin{array}{l}1.6(0.3) \\
13.0 \\
(10.5)\end{array}$ \\
\hline $\begin{array}{l}\text { Patients with nocturnal } \\
\text { awakenings }(\%)\end{array}$ & 63 & 70 & 65 & 56 & 61 & 63 & 41 & 61 & 65 & 65 & 62 & 62 \\
\hline
\end{tabular}

†In all studies the same $0-3$ scale was used to assess symptoms $(0=$ no symptoms, $1=$ mild symptoms, $2=$ moderate symptoms, and $3=$ severe symptoms $)$. Symptom scores were measured in the week preceding randomisation to study treatment and were recorded by patients on a diary card.

$\ddagger$ Peak flow variability $(\%)=\mid$ PM PEF-AM PEF $\mid \times 100$ Average of AM \& PM PEF

Morning and evening peak expiratory flows (PEF) were measured before $\beta_{2}$ agonist use and recorded by patients on a diary card. Baseline values were determined in the week preceding randomisation to study treatment.

zafirlukast $20 \mathrm{mg}$ twice daily compared with placebo; target population-steroid-naive patients with mild to moderate asthma who were appropriate for chronic asthma therapy. The data used in this integrated analysis came from the complete safety and efficacy databases which included all patients who received at least one treatment dose. Patients were recruited and studied between January 1993 and August 1996. All trials were multicentre studies; trials A, B, C, and $\mathrm{E}$ were conducted in North America, and trial $\mathrm{D}$ was a multinational study conducted in Europe. All subjects gave their written informed consent and the trials were conducted in accordance with the Declaration of Helsinki and Guidelines on Good Clinical Practice.

Trial A was a dose ranging trial comparing placebo with zafirlukast $4,10,20,40$ and $80 \mathrm{mg}$ twice daily and $40 \mathrm{mg}$ once a day. ${ }^{11} \mathrm{In}$ this analysis we included only data from subjects who received zafirlukast $20 \mathrm{mg}$ twice daily or placebo. Results of the other four trials which compared zafirlukast $20 \mathrm{mg}$ twice daily with placebo were reported by Fish et $a l^{12}$ (B), Nathan et $a l^{14}$ (C), Holgate et $a l^{15}$ (D), and Nathan et $\mathrm{l}^{13}$ (E). In all these trials the primary outcome measured was asthma symptoms.

\section{PATIENT CHARACTERISTICS}

Male and female patients, both non-smokers or ex-smokers who had not smoked for at least six months and had less than a 10 pack year history (packs/year $\times$ number of years of smoking), with a documented clinical history of asthma were eligible for recruitment. All patients were aged $\geqslant 12$ years and women of childbearing potential were required to practise an effective method of birth control throughout the trial. Trial A included only patients who were aged between 18 and 65 years, and trial D excluded patients aged more than 65 years.

In all studies patients were required to demonstrate reversible airway disease as shown by either a positive histamine or methacholine challenge test within six months of screening or $\mathrm{a} \geqslant 15 \%$ increase in $\mathrm{FEV}_{1}$ or PEF after use of an inhaled bronchodilator. (A positive histamine or methacholine challenge was one in which the provocative concentration that decreased the $\mathrm{FEV}_{1}$ by $20 \%$ was $8 \mathrm{mg} / \mathrm{ml}$ or the provocative dose that decreased the $\mathrm{FEV}_{1}$ by $20 \%$ was < 2 mg.) Patients in trials A, B, and C were required to have an $\mathrm{FEV}_{1} \geqslant 55 \%$ of the predicted value at entry, and patients in trial $\mathrm{E}$ were required to have an $\mathrm{FEV}_{1}$ of $45-80 \%$ of the predicted value. The protocol of trial $\mathrm{D}$ did not specify entry criteria for $\mathrm{FEV}_{1}$, but patients' lung function and symptom levels had to be consistent with definitions of step 2 in international treatment guidelines. ${ }^{16}$

In trials $\mathrm{A}, \mathrm{B}, \mathrm{D}$, and $\mathrm{E}$ patients were maintained on "as required" $\beta_{2}$ agonists alone at entry to the trial. In trial $\mathrm{C}$ patients taking oral theophylline were permitted to enter the trial if the medication was withdrawn prior to or on entry to the one week placebo run in period before randomisation. None of the patients were using oral or inhaled corticosteroids or long acting $\beta_{2}$ agonists at entry to the trial.

Patient characteristics at the time of randomisation to double blind treatment are summarised in table 1. Patients were generally well matched between treatment groups and across trials, with similar disease profiles. Patients in trial D had slightly milder disease as shown by lower mean daytime asthma symptoms scores, fewer mean nocturnal awakenings, and higher mean percentage predicted $\mathrm{FEV}_{1}$ values. All patients in the combined data set had mild to moderately severe, persistent asthma according to the criteria defined in national and international guidelines. ${ }^{16-18}$

\section{DATA EXTRACTION}

In all trials investigators recorded (on case report forms) health care contacts and changes in asthma treatment at the time of withdrawal or scheduled or unscheduled visits. The data from these forms were subsequently validated by data management personnel from Zeneca Pharmaceuticals and entered into computerised databases before the blind was broken.

The data sets analysed included all patients randomised to treatment with either zafirlukast $(n=972)$ or placebo $(n=692)$ from the five trials (full analysis data set or intention-to-treat approach). The 2:1 randomisation allocation 
Table 2 Integrated analysis comparing the number of patients who had asthma exacerbations according to three definitions among patients who received zafirlukast $20 \mathrm{mg}$ twice daily or placebo

\begin{tabular}{lllllll}
\hline Exacerbations that: & $\begin{array}{l}\text { Zafirlukast } \\
(n=972)\end{array}$ & $\begin{array}{l}\text { Placebo } \\
(n=692)\end{array}$ & Odds ratio & $\begin{array}{l}\text { 95\% confidence } \\
\text { interval }\end{array}$ & p value & $\begin{array}{l}\text { p value } \\
\text { (Breslow-Day test) }\end{array}$ \\
\hline Led to withdrawal & $26(2.7)$ & $38(5.5)$ & 0.45 & 0.26 to 0.76 & 0.003 & 0.833 \\
Required additional asthma treatment & $33(3.4)$ & $53(7.7)$ & 0.47 & 0.30 to 0.74 & 0.001 & 0.477 \\
Required oral steroid treatment & $31(3.2)$ & $45(6.5)$ & 0.53 & 0.32 to 0.86 & 0.010 & 0.702 \\
\hline
\end{tabular}

Values are no. (\%).

in favour of zafirlukast in trial B largely explains the larger number of patients receiving zafirlukast overall.

Three analyses, corresponding to three different definitions of asthma exacerbations, were performed on these combined data. Because no standard universally accepted definition of asthma exacerbation exists, we chose those that could reliably be applied to our data set and that appeared to be most clinically relevant.

The first analysis included only asthma exacerbations that required withdrawal from the trials according to the individual trial protocols. In trials $\mathrm{A}, \mathrm{B}$, and $\mathrm{C}$ investigators withdrew patients if they had asthma exacerbations that required any of the following: more than one emergency room visit or hospital admission; the addition of regular inhaled corticosteroids; or a short course of rescue inhaled or oral corticosteroids. In trial D these criteria for withdrawal were expanded to include patients with worsening symptoms that were unrelieved with salbutamol as judged by the patient. In trial $\mathrm{E}$ the criteria for withdrawal were the same as described for studies A, B, and $C$ except that a single seven day course of oral prednisolone was permitted without requiring the patient to be withdrawn from the study.

The second analysis of the combined data set counted patients who received prescriptions for additional anti-asthma therapy other than an increased use of short acting $\beta_{2}$ agonist. The third analysis counted patients who required oral corticosteroid rescue therapy. In both of these analyses patients were included irrespective of whether they were withdrawn from the study.

The three different definitions of exacerbations were not mutually exclusive. All patients who required oral corticosteroid rescue therapy were also counted among the patients who received prescriptions for additional antiasthma therapy. Each patient was counted only once in each analysis even if that patient had multiple exacerbations. The rationale for doing this was that exacerbations led to withdrawal in most cases, thereby eliminating the possibility of counting additional exacerbations. Also, the way the data were captured often made it difficult to distinguish between multiple exacerbations and multiple prescriptions of medication for the same exacerbation.

The data for the patients who required oral corticosteroid rescue therapy were stratified by baseline lung function (percentage peak flow variability and percentage predicted $\mathrm{FEV}_{1}$ ) to determine the effect of asthma severity on exacerbations.
STATISTICAL ANALYSIS

Analyses were performed with all trials combined using a continuity corrected CochranMantel-Haenszel test adjusted for the effects of individual trials. ${ }^{19}{ }^{20} \mathrm{p}$ values of $\leqslant 0.05$ from this test were considered to be statistically significant. The Mantel-Haenszel test-based adjusted odds ratio estimator and corresponding 95\% confidence interval (CI) were calculated to estimate the common relative risk across trials of having an asthma exacerbation. To evaluate the consistency of treatment effect across trials, the Breslow-Day test of homogeneity of odds ratios was calculated for each definition of asthma exacerbation. ${ }^{20}$ In addition, for each trial a $\chi^{2}$ test of independence was performed and the odds ratio and Mantel-Haenszel test-based $95 \%$ CI of the odds ratio were calculated.

\section{Results}

The group treated with zafirlukast $20 \mathrm{mg}$ twice daily had a lower risk of experiencing exacerbations leading to trial withdrawal than the placebo group. Overall, 38 patients $(5.5 \%)$ in the placebo group and 26 patients $(2.7 \%)$ in the zafirlukast group were withdrawn because of an asthma exacerbation (odds ratio $=0.45$; $95 \%$ CI 0.26 to $0.76 ; \mathrm{p}=0.003$; table 2 ). This result was consistent across all five trials, as suggested by the non-significant Breslow-Day test of homogeneity of odds ratios $(p=0.833)$. In the largest of the five trials (trial B) the difference between treatments was also significant (odds ratio $=0.47 ; 95 \%$ CI 0.23 to $0.93 ; \mathrm{p}=$ 0.031 ).

The results of the second analysis, which used the definition of an exacerbation as a requirement for additional anti-asthma therapy (other than increased use of short acting $\beta_{2}$ agonist) were that $33(3.4 \%)$ of the group receiving zafirlukast $20 \mathrm{mg}$ twice daily needed additional anti-asthma therapy compared with $53(7.7 \%)$ of the placebo group. The difference between groups in risk of requiring additional anti-asthma treatment was statistically significant (odds ratio $=0.47 ; 95 \%$ CI 0.30 to 0.74 ; $\mathrm{p}=0.001)$ and consistent across trials $(\mathrm{p}=$ 0.477, Breslow-Day test).

The results of the analysis for the need for oral corticosteroids are shown in fig 1 and table 2. Most of the patients $(69 \%)$ who had asthma exacerbations leading to withdrawal from trials A, B, C, and D required short bursts of oral corticosteroids. Trial $\mathrm{E}$ had the lowest number of exacerbations leading to withdrawal (two $(0.9 \%)$ in the zafirlukast group and six $(2.7 \%)$ in the placebo group) but the highest numbers of patients who required treatment with oral corticosteroids $(12(5.2 \%$ in the zafirlukast 


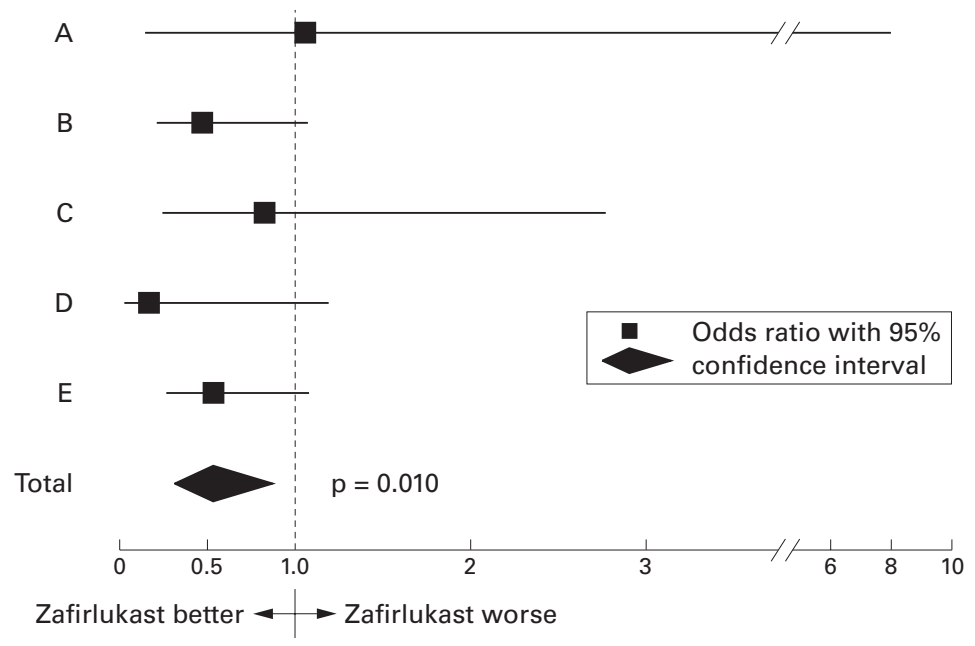

Figure 1 Odds ratio (with 95\% confidence intervals) by trial and overall for asthma exacerbations defined as the need for oral corticosteroids.

group and $21(9.4 \%)$ in the placebo group). This difference is explained by the fact that patients in trial $\mathrm{E}$ may have had more severe disease than the patients in the other trials, and the protocol allowed them to remain in the trial after a single short course of oral corticosteroids. Forty five patients $(6.5 \%)$ receiving placebo compared with $31(3.2 \%)$ receiving zafirlukast $20 \mathrm{mg}$ twice daily required oral corticosteroid rescue (odds ratio $=0.53 ; 95 \%$ CI 0.32 to $0.86 ; p=0.010)$. In all but the smallest of the five trials (trial A) the percentage of patients requiring oral corticosteroid rescues was less in the zafirlukast group

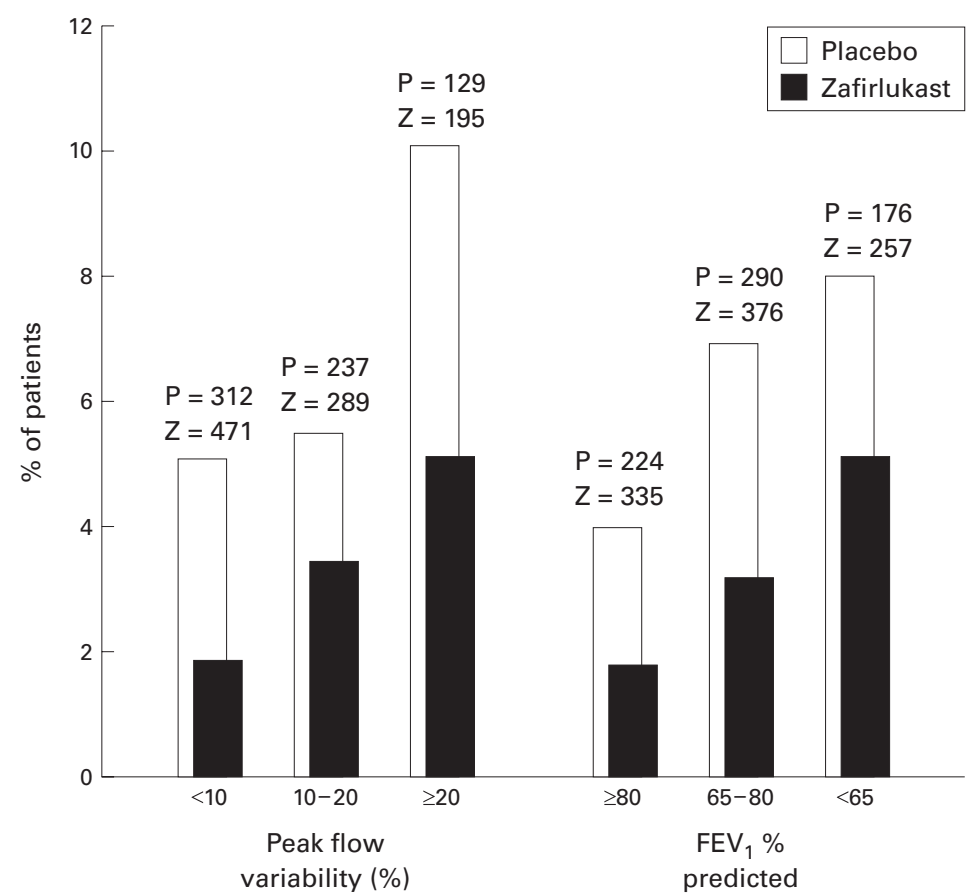

Figure 2 Percentage of patients within each stratified group (stratification by baseline \% peak flow variability * or percentage predicted FEV,) who had asthma exacerbations defined as the need for oral corticosteroids. Numbers above each set of columns are the numbers of patients at risk in each stratified group $(Z=z$ afirlukast, $P=$ placebo $)$. Odds ratios and $95 \%$ CI for peak flow variability subgroups were: $<10 \%, O R=0.40,95 \% C I$ 0.17 to $0.93 ; 10-20 \%, O R=0.70,95 \%$ CI 0.21 to $2.34 ; \geqslant 20 \%, O R=0.56,95 \%$ CI 0.20 to 1.56. Odds ratios and $95 \%$ CI for FEV percentage predicted subgroups were: $<65 \%, O R=0.70,95 \%$ CI 0.24 to $2.02 ; 65-80 \%$, OR $=0.48,95 \%$ CI 0.22 to 1.05 ; $\geqslant 80 \%, O R=0.43,95 \%$ CI 0.12 to 1.55$)$. ${ }^{\star}$ See footnote to table 1 . than in the placebo group. (In trial A only two patients in each treatment group required oral corticosteroids.) Thus, there was little evidence that this treatment effect was not consistent across trials ( $p=0.702$, Breslow-Day test).

In total, 102 patients had an asthma exacerbation that met at least one of the three definitions $(39(4.0 \%)$ on zafirlukast, $63(9.1 \%)$ on placebo). Eighty six (84.3\%) of these patients required the addition of another asthma therapy which included oral corticosteroids in 76 of 86 cases $(88.4 \%)$. Other asthma therapies included inhaled corticosteroids in 19 patients $(22.1 \%)$ and cromones or theophylline in 16 $(18.6 \%)$; some patients received more than one medication. Most of the patients who required additional asthma medications $(n=48,55.8 \%)$ were withdrawn for worsening asthma, which was consistent with the trial protocols. Only 16 patients (six zafirlukast, 10 placebo) were withdrawn for worsening asthma without having received a prescription for an additional asthma medication.

Figure 2 shows the results of stratifying the number of patients who required oral corticosteroid rescue by baseline asthma severity, characterised by percentage predicted $\mathrm{FEV}_{1}$ or peak flow variability. The percentage of patients experiencing exacerbations increased with increasing asthma severity; however, zafirlukast provided a similar degree of protection across all asthma severities.

A total of 167 patients $(17.2 \%)$ in the zafirlukast group and $139(20.1 \%)$ in the placebo group withdrew from the trials before completion. Other than asthma exacerbations, the reasons for withdrawal were similar in the two groups. Twenty one patients $(2.2 \%)$ in the zafirlukast group and $16(2.3 \%)$ in the placebo group withdrew because of adverse events unrelated to asthma and $12.3 \%$ of patients in each group withdrew because of other reasons such as protocol violations, failure to return, and refusal to continue.

\section{Discussion}

Exacerbations of asthma causing unscheduled asthma care, such as admission to hospital or the need to attend a primary care physician or visit an accident and emergency department, are responsible for considerable morbidity and a substantial proportion of the costs of asthma care. ${ }^{21}$ Prevention of asthma exacerbations or improvement of disease control is therefore an important objective in the treatment of chronic asthma. ${ }^{12}$ The results of our analysis of the integrated databases of five trials in mild to moderate steroid-naive asthmatics indicate that, compared with placebo, zafirlukast $20 \mathrm{mg}$ twice daily significantly reduced and more than halved the risk of asthma exacerbations leading to withdrawal from clinical trials or the need for further treatment interventions including oral corticosteroid rescue.

The analysis method we used is a widely accepted way to reach a general conclusion about a particular question which had not been adequately addressed in individual studies due to a lack of statistical power. ${ }^{22}$ In our trials with patients who had mild to moderate asthma the 
incidence of asthma exacerbations was low, as shown by the fact that only $5.5 \%$ of patients receiving placebo withdrew because of asthma exacerbation during the 13 week trial period. By analysing all the data from trials of similar design, we were able to estimate the effect of zafirlukast in a dose of $20 \mathrm{mg}$ twice daily on the risk of exacerbations in patients with mild to moderate asthma. By using the complete 13 week study data set we were able to eliminate publication bias, increase the accuracy of the estimate, and apply different definitions for additional analyses. Although the trials had some minor differences in subject selection and study design, the results were remarkably homogenous. This analysis supports the results of a previous six week trial in patients with more severe asthma (mean $\mathrm{FEV}_{1}$ 66\% predicted) in which $2 \%$ of patients treated with zafirlukast $20 \mathrm{mg}$ twice daily withdrew because of failure of asthma treatment compared with $10 \%$ of patients receiving placebo. ${ }^{11}$

Exacerbations resulting in withdrawal were not analysed as the primary efficacy end point in any of the five trials. However, these data were collected prospectively and investigators and patients were blinded as to treatment. The investigators used their own discretion and judgement as they would have in a routine clinical practice and were not led by preset symptom or pulmonary function limits to make these clinical judgements. Fabbri et $a \bar{l}$ used similar exacerbation data to demonstrate a lower exacerbation rate in patients treated with fluticasone propionate than in those treated with beclomethasone dipropionate in moderate to severe steroid-dependent asthma.

We also defined asthma exacerbations as the need to treat patients with additional asthma therapy including oral corticosteroids. This definition of asthma exacerbations has been employed in several studies that have established the usefulness of inhaled corticosteroid therapy. ${ }^{45}$ In the Formoterol and Corticosteroids Establishing Therapy (FACET) $\operatorname{trial}^{7}$ one of the definitions of severe exacerbations used was the requirement for oral corticosteroids. Additionally, the 5-lipoxygenase inhibitor zileuton and the leukotriene receptor antagonist montelukast have been shown to decrease asthma exacerbations as defined by the need for corticosteroid treatment. In a 13 week placebo controlled trial a retrospective analysis of a subpopulation of patients with more severe asthma $\left(\mathrm{FEV}_{1}<50 \%\right.$ predicted) indicated that zileuton significantly reduced the need for oral corticosteroid therapy compared with placebo. ${ }^{23}$ In a further prospective six month trial ${ }^{24}$ zileuton $600 \mathrm{mg}$ four times daily significantly decreased the need for oral corticosteroid therapy by more than half compared with placebo in patients whose baseline $\mathrm{FEV}_{1}$ was $62 \%$ of predicted. In a large placebo controlled trial in patients with a mean baseline $\mathrm{FEV}_{1}$ of $67 \%$ predicted, $6.9 \%$ of the patients in the group receiving $10 \mathrm{mg}$ montelukast required oral corticosteroid rescues compared with $9.6 \%$ of patients in the placebo group, although the difference was not statistically significant. ${ }^{25}$ More recently, montelukast and beclomethasone dipropionate were compared with placebo in 895 patients whose mean baseline $\mathrm{FEV}_{1}$ was $65 \%$ predicted. $^{26}$ The need for rescue oral corticosteroids or an unscheduled physician or hospital visit was significantly decreased by $63 \%$ in the beclomethasone group and $43 \%$ in the montelukast group.

Although these trials employed definitions of asthma exacerbations that were similar to ours, the rate of exacerbations for the placebo groups in these trials was higher than that in our trial population in which the patients had milder asthma (mean $\mathrm{FEV}_{1} \quad 75 \%$ predicted). Nevertheless, with the increased statistical power afforded by the integrated analysis, we were able to demonstrate a statistically significant approximate halving in the risk of having an exacerbation requiring oral corticosteroid therapy for patients treated with zafirlukast $20 \mathrm{mg}$ twice daily. The robustness of the estimate of effect with zafirlukast compared with placebo is further supported by the consistency of effect across the subgroups of patients stratified by severity of airway obstruction and degree of airway instability at baseline.

Wider definitions of asthma exacerbations other than those we used have included prospectively defined increases in symptoms or $\beta_{2}$ agonist use or a decrease in pulmonary function occurring on at least two days. ${ }^{72} 28$ Noonan et $a l^{28}$ reported that fewer patients $(47.1 \%)$ in a combined group of patients treated with montelukast 10 or $50 \mathrm{mg}$ per day had such exacerbations compared with placebo $(69.6 \%)$, and Reiss et $a l^{25}$ reported a statistically significant $31 \%$ reduction in exacerbation days with montelukast $10 \mathrm{mg}$ per day compared with placebo. In these studies asthma exacerbations were defined by days with increased symptoms or decreased lung function. Using such definitions, asthma exacerbations can be found to be more prevalent, thereby increasing the statistical power of individual studies to detect the beneficial effects of treatment. However, these definitions tend to define relatively mild exacerbations and their relationship to exacerbations of importance in clinical practice is not clear.

Our analyses confirm that zafirlukast monotherapy in steroid-naive asthmatic patients can reduce the risk of asthma exacerbations by half compared with placebo, regardless of the definition of exacerbation that is chosen. Further studies will be needed to confirm these results in patients with more severe asthma and in patients who are receiving concomitant asthma treatments. The FACET trial ${ }^{7}$ showed that, in patients receiving 200 and $800 \mu \mathrm{g} /$ day inhaled budesonide, the addition of the long acting $\beta_{2}$ agonist formoterol reduced severe asthma exacerbations by $26 \%$ compared with placebo. In a four week study of patients with persistent asthma, $80 \%$ of whom were on concurrent corticosteroids, zafirlukast and the long acting $\beta_{2}$ agonist salmeterol had a similar effect on exacerbations. ${ }^{29}$ Further studies are needed to evaluate the effectiveness of zafirlukast compared with long acting $\beta_{2}$ agonists and other established treatment. 
In conclusion, the ability of the leukotriene receptor antagonist zafirlukast to halve the risk of having asthma exacerbations that require further interventions by clinicians represents an important clinical property of this drug. Compared with placebo, zafirlukast $20 \mathrm{mg}$ twice daily reduces the incidence of asthma exacerbations leading to withdrawal from treatment and the need for rescue oral corticosteroid therapy in patients with mild to moderate asthma.

We wish to thank Barbara Murray for assistance with writing and Susy Hassall for technical assistance.

Funding: Research funding was supplied through research grants from Zeneca Pharmaceuticals, USA and Zeneca Limited, UK.

1 Barnes NC. Efficacy and effectiveness in treatment of asthma. Eur Respir Rev 1995;30:284-7.

2 Society of Pharmaceutical Medicine Expert Panel Report. Clinical development of drugs against asthma. 7 Pharm Med 1993;3:115-253.

3 Kerrebijn KF, Van Essen-Zandvliet EEM, Neigens HJ. Effect of long-term treatment with inhaled corticosteroids and beta-agonists on bronchial responsiveness in asthmatic children. F Allergy Clin Immunol 1987;79:653-9.

4 Haahtela $T$, Jarvinen $M$, Kava T, et al. Comparison of a $\beta_{2}$ agonist terbutaline with an inhaled steroid in newly detected asthma. N Engl F Med 1991;325:388-92.

5 Fabbri L, Burge PS, Croonenborgh L, et al. Comparison of fluticasone propionate with beclomethasone dipropionate in moderate to severe asthma treated for one year. International Study Group. Thorax 1993;48:817-23.

6 Chervinsky P, van As A, Bronsky EA, et al. Fluticasone propionate aerosol for the treatment of adults with mild to moderate asthma. The Fluticasone Propionate Asthma moderate asthma. The Fluticasone Propionate Asth

7 Pauwels RA, Lofdahl CG, Postma DS, et al. Effect of inhaled formoterol and budesonide on exacerbations of inhaled formoterol and budesonide on exacerbations of asthma. Formoterol and Corticosteroids Establishing Therapy (FACET) Inte

8 Taylor IK, O'Shaughnessy KM, Fuller RW, et al. Effect of cysteinyl-leukotriene receptor antagonist ICI 204,219 on allergen-induced bronchoconstriction and airway hyperreactivity in atopic subjects. Lancet 1991;337:690-4

9 Finnerty JP, Wood-Baker R, Thomson H, et al. Role of leukotrienes in exercise-induced asthma: inhibitory effect of ICI 204,219, a potent leukotriene $\mathrm{D}_{4}$ receptor antagonist. Am Rev Respir Dis 1992;145:746-9.

10 Drazen JM, O’Brien J, Sparrow D, et al. Recovery of leukotriene $\mathrm{E}_{4}$ from the urine of patients with airway obstruction. Am Rev Respir Dis 1992;146:104-8.

11 Spector SL, Smith LJ, Glass M. Effects of 6 weeks of therapy with oral doses of ICI 204,219, a leukotriene D receptor antagonist, in subjects with bronchial asthma. receptor antagonist, in subjects with bronchial asthma. Med 1994;150:618-23.
12 Fish JE, Kemp JP, Lockey RF, et al. Zafirlukast for symptomatic mild-to-moderate asthma: a 13-week multicenter tudy. The Zafirlukast Trialists Group. Clin Ther 1997;19: $675-90$

13 Nathan RA, Bernstein JA, Bielory L, et al. Zafirlukast improves asthma symptoms and quality of life in patients with moderate airflow obstruction. F Allergy Clin Immunol 1998;102:935-42.

14 Nathan RA, Minkwitz MC, Bonuccelli CM. Two first-line therapies in the treatment of mild asthma: use of peak flow variability as a predictor of effectiveness. Ann Allergy Asthma Immunol 1999;82:497-503.

15 Holgate ST, Anderson KD, Rodgers EM. Comparison of 'Accolate' (zafirlukast) with sodium cromoglycate in mild to moderate asthmatic patients (abstract). Allergy 1995; 50(Suppl 26):319-20.

16 Global Initiative for Asthma. Pocket guide for asthma management and prevention. NIH Publication No. 96-3659B. Bethesda, MD: National Heart, Lung, and Blood Institute; 1995.

17 National Heart, Lung, and Blood Institute. Executive summary: guidelines for the diagnosis and management of asthma. NIH Publication No. 91-3042A. Bethesda, MD: National Asthma Education Program, US Department of Health and Human Services, Public Health Service, 1991.

18 Ayres JG, Barnes PJ, Bellamy D, et al. British guidelines on asthma management: 1995 review and position statement. Thorax 1997;52(Suppl 1):S1-21.

9 Agresti A. Categorical data analysis. New York: John Wiley, 1990: $230-2$.

20 SAS/STAT User's Guide, Vol 1. 4th ed. Cary, NC: SAS Institute Inc, 1990: 876-7.

21 Weiss KB, Gergen PJ, Hodgson TA An economic evaluation of asthma in the United States. $N$ Engl $7 \mathrm{Med}$ 1992;326:862-6.

22 Senn S. Statistical issues in drug development. Chichester, UK: John Wiley, 1997: 219-35.

23 Israel E, Cohn J, Dubé LM, et al. Effect of treatment with zileuton, a 5-lipoxygenase inhibitor, in patients with asthma. A randomized controlled trial. F Am Med Assoc 1996;275:931-6.

24 Liu MC, Dubé LM, Lancaster J. Acute and chronic effects of a 5-lipoxygenase inhibitor in asthma: a 6-month randomized multicenter trial. F Allergy Clin Immunol 1996; 98:859-71.

25 Reiss TF, Chervinsky P, Dockhorn RJ, et al. Montelukast, a once-daily leukotriene receptor antagonist, in the treatment of chronic asthma: a multicenter, randomized, doubleblind trial. Montelukast Clinical Research Study Group. Arch Intern Med 1998;158:1213-20.

26 Malmstrom K, Rodriguez-Gomez G, Guerra J, et al. Oral montelukast, inhaled beclomethasone, and placebo for chronic asthma. A randomized, controlled trial. Ann Intern Med 1999;130:487-95.

27 Wilding P, Clark M, Coon JT, et al. Effect of long term treatment with salmeterol on asthma control: a double blind, randomised crossover study. BMF 1997;314:1441-6.

28 Noonan MJ, Chervinsky P, Brandon M, et al. Montelukast, a potent leukotriene receptor antagonist, causes doserelated improvements in chronic asthma. Montelukast Asthma Study Group. Eur Respir F 1998;11:1232-9.

29 Busse W, Nelson $\mathrm{H}$, Wolfe J, et al. Comparison of inhaled salmeterol and oral zafirlukast in patients with asthma. $\mathcal{F}$ Allergy Clin Immunol 1999;103:1075-80. 